\title{
A highly efficient and convergent synthesis of a hexasaccharide, a dimer of the repeating unit of the antigen $\mathrm{O} 2$ polysaccharide of Stenotrophomonas maltophilia
}

\author{
Wei Wang, Fanzuo Kong* \\ Research Center for Eco-Environmental Sciences, Academia Sinica, PO Box 2871, \\ Beijing 100085, People's Republic of China
}

Received 15 August 1998; revised 25 November 1998; accepted 1 December 1998

\begin{abstract}
A highly efficient and convergent synthesis of a hexasaccharide, which is a dimer of the repeating unit of the antigen $\mathrm{O} 2$ polysaccharide of Stenotrophomonas maltophilia, was achieved via coupling of 2,3,4-tri- $O$-acetyl- $\alpha$-L-xylopyranosyl bromide with the tetrasaccharide, allyl 4- $O$-\{3- $O$-[4- $O$-(3,4-di- $O$-benzoyl- $\alpha$-L-rhamnopyranosyl)-2,3,6tri- $O$-benzoyl- $\alpha$-D-mannopyranosyl]-4-benzoyl- $\alpha$-L-rhamnopyranosyl $\}-2,3,6$-tri- $O$-benzoyl- $\alpha$-D-mannopyranoside (18) by the Koenigs-Knorr method followed by deacylation. Compound $\mathbf{1 8}$ was readily prepared from the coupling of the disaccharide trichloroacetimidate, 4- $O$-(2- $O$-acetyl-3,4-di- $O$-benzoyl- $\alpha$-L-rhamnopyranosyl)-2,3,6-tri- $O$-benzoyl$\alpha$-D-mannopyranosyl trichloroacetimidate $(8)$ with the disaccharide acceptor, allyl 4- $O$ - $(2-O$-acetyl-4- $O$-benzoyl- $\alpha$-Lrhamnopyranosyl)-2,3,6-tri- $O$-benzoyl- $\alpha$-D-mannopyranoside (16), and both $\mathbf{8}$ and $\mathbf{1 6}$ were prepared via the trichloroacetimidate method from simple starting materials. The sole use of acyl protecting groups substantially

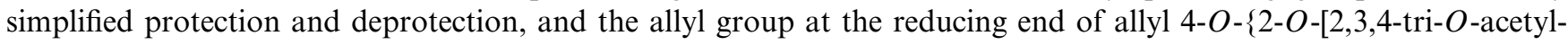
$\beta$-L-xylopyranosyl]-3- $O$-[4- $O$-(2- $O$-(2,3,4-tri- $O$-acetyl- $\beta$-L-xylopyranosyl)-3,4-di- $O$-benzoyl- $\alpha$-L-rhamnopyranosyl)2,3,6-tri- $O$-benzoyl- $\alpha$-D-mannopyranosyl]-4- $O$-benzoyl- $\alpha$-L-rhamnopyranosyl $\}-2,3,6$-tri- $O$-benzoyl- $\alpha$-D-mannopyranoside 19 allowed further chemical transformation. (C) 1999 Elsevier Science Ltd. All rights reserved.
\end{abstract}

Keywords: Synthesis of hexasaccharide; O2 antigen of Stenotrophomonas maltophilia; Glycosylation

\section{Introduction}

The organism known as Stenotrophomonas (Xanthomonas or Pseudomonas) maltophilia [1] is a free-living organism which is commonly present in clinical specimens. The organism has a growing reputation as an opportunistic pathogen, particularly as an agent of nosocomial infection, and a potential threat to the

* Corresponding author. Fax: + 86-10-62923563.

E-mail address: fzkong@mail.rcees.ac.cn (F. Kong) cystic fibrosis population, which is partly based on the multidrug resistance of many strains [2]. A polysaccharide consisting of $[(-3) \mathrm{L}-\mathrm{Xyl} p(\beta 1-2) \mathrm{L}-\mathrm{Rha} p(\alpha 1-4) \mathrm{D}-\operatorname{Man} p(\alpha 1-)]_{n}$ was isolated from the lipopolysaccharide (LPS) present in the reference strain for Stenotrophomonas maltophilia serogroup $\mathrm{O} 2$ by Winn and Wilkinson in 1997 [2]. We report here a facile synthesis of a hexasaccharide $\mathbf{2 0}$, which is a dimer of the repeating unit of the antigen $\mathrm{O} 2$ polymer. 


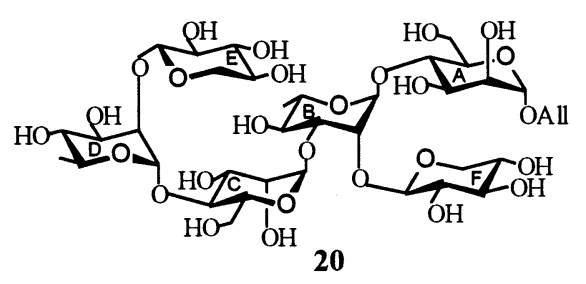

\section{Results and discussion}

Retrosynthetic analysis indicated that building a tetrasaccharide $\mathrm{ABCD}$ block first and then coupling the L-xylose unit at the B-2 and D-2 positions would be an effective and simple route, especially since the $\mathrm{ABCD}$ block can be readily constructed from two disaccharide blocks $\mathrm{AB}$ and $\mathrm{CD}$. Thus, the glycoside acceptor 1, allyl 2,3,6-tri- $O$-benzoyl- $\alpha$-D-mannopyranoside, was obtained from allyl $\alpha$-Dmannopyranoside [3] through selective tribenzoylation with benzoyl chloride in pyridine under the conditions designated for selective benzoylation of trehalose [4] (Scheme 1). The ${ }^{1} \mathrm{H}$ NMR of 1 gave $\mathrm{H}-4$ as an upfield triplet $(\delta$ $4.3 \mathrm{ppm}$ ), a clear indication of a free $\mathrm{OH}$ hydroxyl. For the synthesis of disaccharide $\mathbf{6}$,

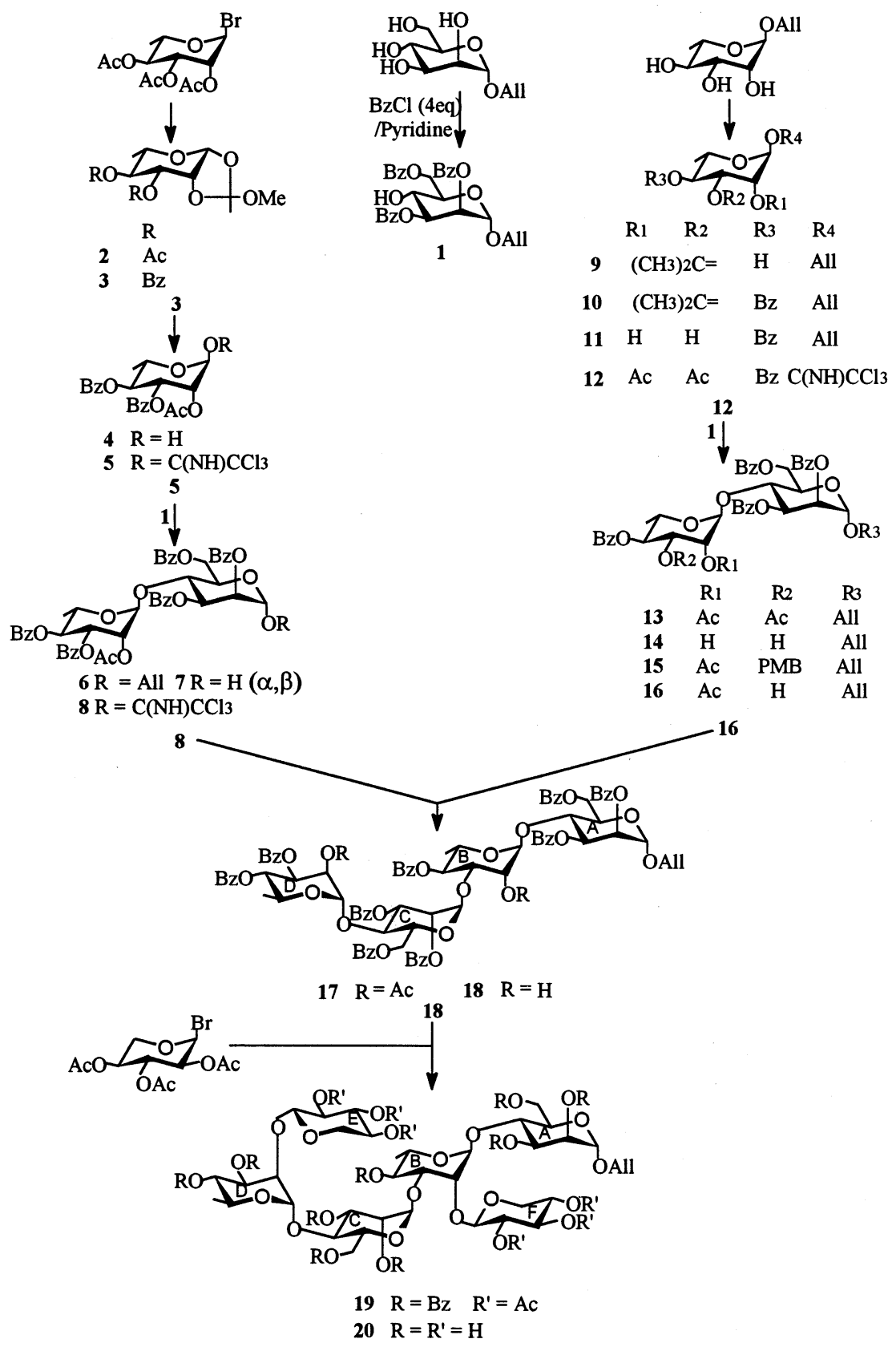

Scheme 1. 
an orthoester intermediate was used. Treatment of 'acetobromorhamnose' with $\mathrm{MeOH}$ in the presence of 2,4-lutidine $/{ }^{t} \mathrm{Bu}_{4} \mathrm{NBr}$ instead of sym-collidine $/{ }^{t} \mathrm{Bu}_{4} \mathrm{NBr}$ [5] gave orthoester 2 in high yield. Deacetylation of $\mathbf{2}$, followed by benzoylation, gave orthoester $\mathbf{3}$ in quantitative yield. Hydrolysis of 3 in $70 \%$ AcOH solution under the conditions for the transformation of an orthoester to an acetate of an axial hydroxyl group [6] selectively afforded 2-Oacetyl-3,4-di- $O$-benzoyl- $\alpha, \beta$-L-rhamnopyranose 4 with O-1 free, which was converted to the trichloroacetimidate glycosyl donor $\mathbf{5}$ on treatment with $\mathrm{CNCCl}_{3} / \mathrm{DBU}$ [7]. Glycosylation [8] of the acceptor 1 with the trichloroacetimidate donor 5 was promoted with $\mathrm{Me}_{3} \mathrm{SiOTf}$, affording the disaccharide $\mathbf{6}$ in a high yield ( $>90 \%$ ). Deallylation [9] of 6 with palladium chloride in $\mathrm{MeOH}$ followed by treatment with trichloroacetonitrile in the presence of $\mathrm{DBU}$ afforded the disaccharide donor $\mathbf{8}$, which was directly used in the next coupling reaction. The disaccharide acceptor $\mathbf{1 6}$ was prepared starting from allyl $\alpha$-L-rhamnopyranoside [10]. Isopropylidenation of allyl rhamnopyranoside (giving 9) followed by benzoylation afforded allyl 4- $O$-benzoyl-2,3- $O$-isopropylidene- $\alpha-\mathrm{L}-$ rhamnopyranoside (10) [11]. Hydrolysis of $\mathbf{1 0}$ followed by acetylation, then deallylation, and subsequent treatment with trichloroacetonitrile in the presence of DBU afforded the required donor 12. $\mathrm{Me}_{3} \mathrm{SiOTf}$-promoted glycosylation of the acceptor $\mathbf{1}$ with the trichloroacetimidate $\mathbf{1 2}$ gave the disaccharide 13 in $86 \%$ yield. Selective deacetylation [12] of 13 with $\mathrm{CH}_{3} \mathrm{COCl}-\mathrm{MeOH}$ gave the disaccharide 16 as the major product $\left(R_{f} 0.65,2: 1\right.$ petroleum ether-EtOAc) in $65 \%$ yield, which was easily separated from a minor product $\left(R_{f}\right.$ $0.29,2: 1$ petroleum ether-EtOAc) and from the starting material $13 \quad\left(R_{f} \quad 0.82,2: 1\right.$ petroleum ether-EtOAc). To confirm the selective 3'-deacetylation, an unambiguous, alternative synthesis of $\mathbf{1 6}$ from 13 was carried out. Thus complete deacetylation of $\mathbf{1 3}$ with $\mathrm{CH}_{3} \mathrm{COCl}-\mathrm{MeOH}$ after extended reaction time gave allyl 4-O-(4-O-benzoyl- $\alpha$-L-rhamnopyranosyl)-2,3,6-tri- $O$ - benzoyl - $\alpha$-D - mannopyranoside $14\left(R_{f} 0.29,2: 1\right.$ petroleum etherEtOAc). Selective 3'-O-4-methoxybenzylation via the corresponding dibutyltin complex intermediate [13] followed by acetylation af- forded 15, and subsequent selective 3'-deprotection with DDQ [14] furnished a product identical to that obtained from direct selective $3^{\prime}$-deacetylation of $\mathbf{1 3}$, as indicated from TLC $\left(R_{f} 0.65,2: 1\right.$ petroleum etherEtOAc) and ${ }^{1} \mathrm{H}$ NMR.

Glycosylation of the disaccharide donor $\mathbf{8}$ with the disaccharide acceptor $\mathbf{1 6}$ in the presence of $\mathrm{Me}_{3} \mathrm{SiOTf}$ afforded the tetrasaccharide $\mathbf{1 7}$ in $75 \%$ yield. The ${ }^{1} \mathrm{H}$ NMR spectrum of $\mathbf{1 7}$ showed two singlets at $\delta 2.26$ and 2.02 (2 $\left.\mathrm{CH}_{3} \mathrm{CO}\right)$, and two doublets at $\delta 0.85$ and 0.72 ( $-\mathrm{CH}_{3}$ of rhamnose), which came from the rhamnose moieties, and four broad singlets at $\delta$ 5.14, 5.08, 5.04 and 4.99 for $\mathrm{H}-1$ of $\mathrm{A}, \mathrm{B}, \mathrm{C}$, and D units. Selective B-2 and D-2 deacetylation of 17 with $\mathrm{CH}_{3} \mathrm{COCl}-\mathrm{MeOH}$ gave tetrasaccharide acceptor $\mathbf{1 8}$ in high yield.

Glycosylation of the acceptor 18 with $2,3,4$ tri- $O$-acetyl- $\alpha$-L-xylopyranosyl bromide [15] promoted by silver triflate gave the final hexasaccharide 19 in good yield. All of the tribenzoylated mannose-containing oligosaccharides, i.e., the disaccharides $6,7, \mathbf{8}, \mathbf{1 4}, \mathbf{1 5}$, and 16, the tetrasaccharides $\mathbf{1 7}$ and $\mathbf{1 8}$, and the hexasaccharide 19, showed the H-6 of rhamnose residues at $\delta 0.61-0.87$ in their ${ }^{1} \mathrm{H}$ NMR spectra. These values differed from the normal region of around $\delta 1.3($ e.g., $\delta 1.31$ for 4 ) and we rationalized that the unusual upfield shifting was the result of shielding by the benzene ring in the tribenzoylated mannose moieties. Deprotection [16] of 19 in $\mathrm{MeOH}-\mathrm{MeONa}$ readily afforded the title hexasaccharide $\mathbf{2 0}$ in quantitative yield.

The allyl hexasaccharide glycoside consisting of the two trisaccharide units can be used not only for bioactivity study, but also for the preparation of glycoconjugates via conjugation to protein carriers. In addition, the designated strategy for the preparation of the hexasaccharide can be extended to the synthesis of higher oligosaccharides corresponding to the antigen $\mathrm{O} 2$ polymer.

\section{Experimental}

General methods. - Melting points were determined with a 'Mel-Temp' apparatus and optical rotations were determined with a Perkin-Elmer model 241-MC automatic po- 
larimeter for solutions in a 1-dm, jacketed cell. ${ }^{1} \mathrm{H}$ NMR spectra were recorded with Varian XL-400 and Varian XL-200 spectrometers, for solutions in $\mathrm{CDCl}_{3}$ with $\mathrm{Me}_{4} \mathrm{Si}$ as the internal standard. Chemical shifts are expressed in ppm downfield from the internal $\mathrm{Me}_{4} \mathrm{Si}$ absorption. Mass spectrometry was conducted on a JDS-D 3005 mass spectrometer using a direct-insertion technique to introduce the sample. Thin-layer chromatography (TLC) was performed on silica gel $\mathrm{HF}$, detection being effected by charring with $30 \%(\mathrm{v} / \mathrm{v})$ sulfuric acid in methanol or sometimes by UV detection. Column chromatography was conducted by elution of a column of silica gel (100-200 mesh) using EtOAc-petroleum ether (bp $60-90^{\circ} \mathrm{C}$ ) as the solvent. Solutions were concentrated at a temperature $<60^{\circ} \mathrm{C}$ under diminished pressure. Analytical LC was performed with a Gilson HPLC consisting of a pump (model 306), a stainless steel column packed with silica gel (Spherisorb $\mathrm{SiO}_{2}, 10 \times$ $300 \mathrm{~mm}$ or $4.6 \times 250 \mathrm{~mm}$ ), a differential refractometer (132-RI Detector), and a UV-Vis detector (model 118), and EtOAc-petroleum ether (bp $60-90{ }^{\circ} \mathrm{C}$ ) was used as the solvent at a flow rate of $1-4 \mathrm{~mL} / \mathrm{min}$.

Allyl 2,3,6-tri-O-benzoyl- $\alpha$-D-mannopyranoside (1). - Freshly distilled benzoyl chloride (11 mL, $92 \mathrm{mmol}$ ) was added dropwise to a solution of allyl $\alpha$-D-mannopyranoside $(5.0 \mathrm{~g}$, $23 \mathrm{mmol})$ in dry pyridine $(200 \mathrm{~mL})$ at $0{ }^{\circ} \mathrm{C}$. The mixture was allowed to warm to room temperature (rt) and was stirred overnight. The reaction was quenched with $\mathrm{MeOH}$ (10 $\mathrm{mL}$ ), the mixture was concentrated, and the residual solution was poured into ice-water, extracted with $\mathrm{CH}_{2} \mathrm{Cl}_{2}(80 \mathrm{~mL})$ and washed sequentially with $\mathrm{N} \mathrm{HCl}(50 \mathrm{~mL})$, satd aq $\mathrm{NaHCO}_{3}(50 \mathrm{~mL})$, and aq $\mathrm{NaCl}(50 \mathrm{~mL})$. The aq phases were re-extracted with $\mathrm{CH}_{2} \mathrm{Cl}_{2}(40$ $\mathrm{mL}$ ), and the combined organic solutions were dried, concentrated, and purified by column chromatography with $1: 1$ petroleum etherEtOAc as the eluent to give the title compound 1 as a colorless syrup $(9.2 \mathrm{~g}, 76 \%$ ); $[\alpha]_{\mathrm{D}}^{20}+1.85^{\circ} \quad\left(c \quad 1.6, \quad \mathrm{CHCl}_{3}\right) ;{ }^{1} \mathrm{H} \quad \mathrm{NMR}$ $\left(\mathrm{CDCl}_{3}\right): \delta 8.20-7.90(\mathrm{~m}, 6 \mathrm{H}, \mathrm{Bz}-\mathrm{H}), 7.70-$ $7.23(\mathrm{~m}, 9 \mathrm{H}, \mathrm{Bz}-\mathrm{H}), 6.08-5.84(\mathrm{~m}, 1 \mathrm{H}$, $\left.\mathrm{CH}_{2}=\mathrm{CH}-\mathrm{CH}_{2}-\right), 5.65\left(\mathrm{dd}, 1 \mathrm{H}, J_{2,3} 2.6 \mathrm{~Hz}\right.$, $\left.J_{3,4} 9.3 \mathrm{~Hz}, \mathrm{H}-3\right), 5.63\left(\mathrm{dd}, 1 \mathrm{H}, J_{1,2} 1.0 \mathrm{~Hz}\right.$,
$\left.J_{2,3} 2.6 \mathrm{~Hz}, \mathrm{H}-2\right), \quad 5.41-5.20$ (m, $2 \mathrm{H}$, $\left.\mathrm{CH}_{2}=\mathrm{CH}-\mathrm{CH}_{2}-\right), 5.07\left(\mathrm{~d}, 1 \mathrm{H}, J_{1,2} 1.0 \mathrm{~Hz}\right.$, $\mathrm{H}-1), 4.89$ (dd, $1 \mathrm{H}, J_{5,6} 4.0 \mathrm{~Hz}, J_{6,6^{\prime}} 12.6 \mathrm{~Hz}$, H-6), $4.66\left(\mathrm{dd}, 1 \mathrm{H}, J_{5,6^{\prime}} 2.1 \mathrm{~Hz}, J_{6,6^{\prime}} 12.6 \mathrm{~Hz}\right.$, H-6'), 4.30 (t, $1 \mathrm{H}, J 9.3 \mathrm{~Hz}, \mathrm{H}-4), 4.38-4.15$ (m, $3 \mathrm{H}, \mathrm{H}-5, \mathrm{CH}_{2}=\mathrm{CH}-\mathrm{CH}_{2}-$ ). Anal. Calcd for $\mathrm{C}_{30} \mathrm{H}_{28} \mathrm{O}_{9}: \mathrm{C}, 67.66 ; \mathrm{H}, 5.30$. Found: $\mathrm{C}$, 67.80; H, 5.36.

3,4-Di-O-acetyl-1,2-O-methoxyethylidene- $\beta$ L-rhamnopyranose (2). - A mixture of 2,3,4tri- $O$-acetyl- $\alpha$-L-rhamnopyranosyl bromide $(2.0 \mathrm{~g}, 5.7 \mathrm{mmol})$, 2,4-lutidine $(0.8 \mathrm{~mL}, 7.2$ mmol), and tetrabutylammonium bromide $(0.8 \mathrm{~g}, 2.4 \mathrm{mmol})$ in $\mathrm{CH}_{2} \mathrm{Cl}_{2}(15 \mathrm{~mL})$ was stirred at $\mathrm{rt}$ for 3-5 min, then $\mathrm{MeOH}$ (anhyd, $0.5 \mathrm{~mL}, 12 \mathrm{mmol})$ was added. TLC (1:1 petroleum ether-EtOAc) indicated that the reaction was complete. The reaction mixture was concentrated and subjected to column chromatography to give the title compound $\mathbf{2}$ $(1.7 \mathrm{~g}, 97 \%)$. Compound 2 gave ${ }^{1} \mathrm{H}$ NMR data identical to those reported in the literature [5b]; mp 80-84 ${ }^{\circ} \mathrm{C} ;[\alpha]_{\mathrm{D}}^{20}+31^{\circ}\left(c 1.0, \mathrm{CHCl}_{3}\right)$; Ref. [5c] mp $84-86^{\circ} \mathrm{C} ;[\alpha]_{\mathrm{D}}^{20}+34.7^{\circ}$.

3,4-Di-O-benzoyl-2-O-acetyl- $\alpha, \beta$-L-rhamnopyranose (4).-Compound 2 (1.5 g, 4.9 mmol) was deacetylated with $\mathrm{MeOH}-\mathrm{Me}-$ $\mathrm{ONa}$, and then benzoylated with $\mathrm{BzCl}-$ pyridine to furnish 3 in quantitative yield. Hydrolysis of 3 with $70 \%$ aq HOAc [6] at $\mathrm{rt}$ furnished $4(1.6 \mathrm{~g}, 3.9 \mathrm{mmol})$ as a colorless syrup in $80 \%$ yield. Flash chromatography (2:1 petroleum ether-EtOAc) of the residue gave an anomeric mixture $(\alpha / \beta, 10 / 1)$; for $\alpha$ anomer: $[\alpha]_{\mathrm{D}}^{20}+63.9^{\circ}\left(c 0.5, \mathrm{CHCl}_{3}\right) ;{ }^{1} \mathrm{H}$ NMR $\left(\mathrm{CDCl}_{3}\right): \delta(\alpha) 7.96,7.85(2 \mathrm{~d}, 4 \mathrm{H}, J 7.6 \mathrm{~Hz}$, $\mathrm{Bz}-\mathrm{H}), 7.56-7.25(\mathrm{~m}, 6 \mathrm{H}, \mathrm{Bz}-\mathrm{H}), 5.81(\mathrm{dd}, 1$ $\left.\mathrm{H}, J_{2,3} 3.4 \mathrm{~Hz}, J_{3,4} 9.8 \mathrm{~Hz}, \mathrm{H}-3\right), 5.57(\mathrm{t}, 1 \mathrm{H}$, $J 9.8 \mathrm{~Hz}, \mathrm{H}-4), 5.52\left(\mathrm{dd}, 1 \mathrm{H}, J_{1,2} 1.5 \mathrm{~Hz}, J_{2,3}\right.$ $3.4 \mathrm{~Hz}, \mathrm{H}-2), 5.31\left(\mathrm{~d}, 1 \mathrm{H}, J_{1,2} 1.5 \mathrm{~Hz}, \mathrm{H}-1\right)$, 4.49-4.31 (m, $1 \mathrm{H}, \mathrm{H}-5), 2.17$ (s, $3 \mathrm{H}$, $\left.\mathrm{CH}_{3} \mathrm{CO}\right), 1.31\left(\mathrm{~d}, 3 \mathrm{H}, J_{5,6} 6.7 \mathrm{~Hz}, \mathrm{CH}_{3}\right)$. Anal. Calcd for $\mathrm{C}_{22} \mathrm{H}_{22} \mathrm{O}_{8}: \mathrm{C}, 63.76 ; \mathrm{H}, 5.35$. Found: C, 63.90; H, 5.35.

Allyl 4-O-(2-O-acetyl-3,4-di-O-benzoyl- $\alpha$-Lrhamnopyranosyl) - 2,3,6-tri-O - benzoyl - $\alpha$ - Dmannopyranoside (6). - Treatment [7] of $\mathbf{4}$ (330 mg, $0.80 \mathrm{mmol}$ ) with trichloroacetonitrile $\left(\mathrm{Cl}_{3} \mathrm{CCN}, 3\right.$ equiv) and 1,8-diazabicyclo[5,4,0]undec-7-ene (DBU, 0.25 equiv) at $0{ }^{\circ} \mathrm{C}$ in anhyd $\mathrm{CH}_{2} \mathrm{Cl}_{2}$ gave the glycoside donor, trichloroacetimidate 5 (370 mg, 72\%) 
after flash chromatography, which was used directly in the glycosylation reaction. A mixture of the donor $5(370 \mathrm{mg}, 0.66 \mathrm{mmol})$ and the acceptor $1(266 \mathrm{mg}, 0.50 \mathrm{mmol})$ in anhyd $\mathrm{CH}_{2} \mathrm{Cl}_{2}(20 \mathrm{~mL})$ containing $4 \AA$ activated molecular sieves $(0.5 \mathrm{~g})$ was stirred under $\mathrm{N}_{2}$ for $1 \mathrm{~h}$ at $\mathrm{rt}$ and then cooled to $-40{ }^{\circ} \mathrm{C}$. Trimethylsilyl triflate $\left(\mathrm{Me}_{3} \mathrm{SiOTf}, 20 \mu \mathrm{L}, 0.2\right.$ equiv) was added and the mixture was stirred under $\mathrm{N}_{2}$ below $-30{ }^{\circ} \mathrm{C}$ for $40 \mathrm{~min}$. TLC showed the starting material had disappeared. Triethylamine $(15 \mu \mathrm{L})$ was added to the reaction solution and the solid was filtered off and washed with $\mathrm{CH}_{2} \mathrm{Cl}_{2}(30 \mathrm{~mL})$. The combined filtrate and washings were washed sequentially with $\mathrm{H}_{2} \mathrm{O}(30 \mathrm{~mL}), \mathrm{N} \mathrm{HCl}(30 \mathrm{~mL})$, satd aq $\mathrm{NaHCO}_{3}(30 \mathrm{~mL})$, and satd aq $\mathrm{NaCl}(30 \mathrm{~mL})$. The aq washings were re-extracted with $\mathrm{CH}_{2} \mathrm{Cl}_{2}(30 \mathrm{~mL})$ and the combined organic solutions were dried and concentrated, and the residue was subjected to flash chromatography with 3:1 petroleum ether-EtOAc as the eluent to give $6(420 \mathrm{mg}, 0.45 \mathrm{mmol})$ in high yield $(90 \%) ;[\alpha]_{\mathrm{D}}^{20}-40.4^{\circ}\left(c 0.6, \mathrm{CHCl}_{3}\right) ;{ }^{1} \mathrm{H}$ NMR $\left(\mathrm{CDCl}_{3}\right): \delta 8.20-7.80(\mathrm{~m}, 10 \mathrm{H}, \mathrm{Bz}-\mathrm{H})$, 7.67-7.24 (m, $15 \mathrm{H}, \mathrm{Bz}-\mathrm{H}), 6.10-5.90$ (m, 1 $\left.\mathrm{H}, \mathrm{CH}_{2}=\mathrm{CH}-\mathrm{CH}_{2}-\right), 5.91\left(\mathrm{dd}, 1 \mathrm{H}, J_{2,3} 3.4\right.$ $\left.\mathrm{Hz}, J_{3.4} 9.5 \mathrm{~Hz}, \mathrm{H}-3\right), 5.65\left(\mathrm{dd}, 1 \mathrm{H}, J_{1,2} 1.5 \mathrm{~Hz}\right.$, $\left.J_{2,3} 3.4 \mathrm{~Hz}, \mathrm{H}-2\right), 5.60\left(\mathrm{dd}, 1 \mathrm{H}, J_{2^{\prime}, 3^{\prime}} 3.2 \mathrm{~Hz}\right.$, $\left.J_{3^{\prime}, 4^{\prime}} 9.8 \mathrm{~Hz}, \mathrm{H}-3^{\prime}\right), 5.41\left(\mathrm{dd}, 1 \mathrm{H}, J_{1^{\prime}, 2^{\prime}} 1.7 \mathrm{~Hz}\right.$, $\left.J_{2^{\prime}, 3^{\prime}} 3.2 \mathrm{~Hz}, \mathrm{H}-2^{\prime}\right), 5.45-5.25\left(\mathrm{~m}, 3 \mathrm{H}, \mathrm{H}-4^{\prime}\right.$, $\left.\mathrm{CH}_{2}=\mathrm{CH}-\mathrm{CH}_{2}-\right), 5.11\left(\mathrm{~d}, 1 \mathrm{H}, J_{1,2} 1.5 \mathrm{~Hz}\right.$, $\mathrm{H}-1), 5.05$ (d, $\left.1 \mathrm{H}, J_{1^{\prime}, 2^{\prime}} 1.7 \mathrm{~Hz}, \mathrm{H}-1^{\prime}\right), 5.00$ (dd, $\left.1 \mathrm{H}, J_{5,6 \mathrm{a}} 1.7 \mathrm{~Hz}, J_{6 \mathrm{a}, 6 \mathrm{~b}} 12.7 \mathrm{~Hz}, \mathrm{H}-6 \mathrm{a}\right), 4.67$ $\left(\mathrm{dd}, 1 \mathrm{H}, J_{5,6 \mathrm{~b}} 3.4 \mathrm{~Hz}, J_{6 \mathrm{a}, 6 \mathrm{~b}} 12.7 \mathrm{~Hz}, \mathrm{H}-6 \mathrm{~b}\right)$, $4.57\left(\mathrm{t}, 1 \mathrm{H}, J_{3,4} 9.5 \mathrm{~Hz}, J_{4,5} 9.5 \mathrm{~Hz}, \mathrm{H}-4\right)$, $4.40-3.94\left(\mathrm{~m}, 4 \mathrm{H}, \mathrm{H}-5,5^{\prime}, \mathrm{CH}_{2}=\mathrm{CH}-\mathrm{CH}_{2}-\right)$, $2.03\left(\mathrm{~s}, 3 \mathrm{H}, \mathrm{CH}_{3} \mathrm{CO}\right), 0.74\left(\mathrm{~d}, 3 \mathrm{H}, J_{5^{\prime}, 6^{\prime}} 6.5\right.$ $\mathrm{Hz}, \mathrm{CH}_{3}$ ). Anal. Calcd for $\mathrm{C}_{52} \mathrm{H}_{48} \mathrm{O}_{16}: \mathrm{C}$, 67.23; H, 5.21. Found: $\mathrm{C}, 67.12 ; \mathrm{H}, 5.32$.

4-O- (2-O-Acetyl-3,4-di-O - benzoyl - $\alpha$ - Lrhamnopyranosyl)-2,3,6-tri-O-benzoyl- $\alpha, \beta$-Dmannopyranose (7).-Deallylation [9] of 6 (400 mg, $0.43 \mathrm{mmol})$ with $\mathrm{PdCl}_{2}(1: 20 \mathrm{~g} / \mathrm{g})$ in $\mathrm{MeOH}(10 \mathrm{~mL})$ at $\mathrm{rt}$ furnished an amorphous solid 7 (320 mg, $0.36 \mathrm{mmol}, 84 \%$ ), presented mostly as $\alpha$ anomer; $[\alpha]_{\mathrm{D}}^{20}-40.4^{\circ}$ (c) 0.7 $\left.\mathrm{CHCl}_{3}\right) ;{ }^{1} \mathrm{H}$ NMR $\left(\mathrm{CDCl}_{3}\right): \delta 8.20-7.80(\mathrm{~m}$, $10 \mathrm{H}, \mathrm{Bz}-\mathrm{H}), 7.68-7.20(\mathrm{~m}, 15 \mathrm{H}, \mathrm{Bz}-\mathrm{H})$, $5.97\left(\mathrm{dd}, 1 \mathrm{H}, J_{2,3} 3.4 \mathrm{~Hz}, J_{3,4} 9.2 \mathrm{~Hz}, \mathrm{H}-3\right)$, $5.67\left(\mathrm{dd}, 1 \mathrm{H}, J_{1,2} 1.9 \mathrm{~Hz}, J_{2,3} 3.4 \mathrm{~Hz}, \mathrm{H}-2\right)$, $5.60\left(\mathrm{dd}, 1 \mathrm{H}, J_{2^{\prime}, 3^{\prime}} 3.2 \mathrm{~Hz}, J_{3^{\prime}, 4^{\prime}} 9.8 \mathrm{~Hz}, \mathrm{H}-3^{\prime}\right)$, $5.45\left(\mathrm{~d}, 1 \mathrm{H}, J_{1,2} 1.9 \mathrm{~Hz}, \mathrm{H}-1\right), 5.43(\mathrm{dd}, 1 \mathrm{H}$, $\left.J_{1^{\prime}, 2^{\prime}} 1.7 \mathrm{~Hz}, J_{2^{\prime}, 3^{\prime}} 3.2 \mathrm{~Hz}, \mathrm{H}-2^{\prime}\right), 5.40(\mathrm{t}, 1 \mathrm{H}, J$ $\left.9.8 \mathrm{~Hz}, \mathrm{H}-4^{\prime}\right), 5.14\left(\mathrm{~d}, 1 \mathrm{H}, J_{1^{\prime} 2^{\prime}} 1.7 \mathrm{~Hz}, \mathrm{H}-1^{\prime}\right)$, $5.06\left(\mathrm{dd}, 1 \mathrm{H}, J_{5,6 \mathrm{a}} 0.7 \mathrm{~Hz}, J_{6 \mathrm{a}, 6 \mathrm{~b}} 12.4 \mathrm{~Hz}\right.$, H-6a), $4.65\left(\mathrm{dd}, 1 \mathrm{H}, J_{5,6 \mathrm{~b}} 2.0 \mathrm{~Hz}, J_{6 \mathrm{a}, 6 \mathrm{~b}} 12.4\right.$ $\mathrm{Hz}, \mathrm{H}-6 \mathrm{~b}), 4.60$ (t, $1 \mathrm{H}, J 9.2 \mathrm{~Hz}, \mathrm{H}-4)$, $4.62-4.51(\mathrm{~m}, 1 \mathrm{H}, \mathrm{H}-5), 4.12-3.96(\mathrm{~m}, 1 \mathrm{H}$, H-5'), 2.07 (s, $\left.3 \mathrm{H}, \mathrm{CH}_{3} \mathrm{CO}\right), 0.78$ (d, $3 \mathrm{H}, J$ $6.2 \mathrm{~Hz}, \mathrm{CH}_{3}$ ). Anal. Calcd for $\mathrm{C}_{49} \mathrm{H}_{44} \mathrm{O}_{16}: \mathrm{C}$, 66.21; H, 4.99. Found: C, 66.14, H, 5.05.

4-O-(2-O-Acetyl-3,4-di-O-benzoyl- $\alpha$-L-rhamnopyranosyl)-2,3,6-tri-O-benzoyl- $\alpha$ - D-mannopyranosyl trichloroacetimidate (8).-Trichloroacetonitrile $\left(\mathrm{Cl}_{3} \mathrm{CCN}, 0.11 \mathrm{~mL}, 3\right.$ equiv) and 1,8-diazabicyclo[5,4,0]undec-7-ene (DBU, $14 \mu \mathrm{L}, 0.25$ equiv) were added to a solution of 7 (310 mg, $0.35 \mathrm{mmol})$ in anhyd $\mathrm{CH}_{2} \mathrm{Cl}_{2}(10$ $\mathrm{mL}$ ) and the mixture was stirred at $0{ }^{\circ} \mathrm{C}$ for 1 $\mathrm{h}$, at the end of which time TLC showed the starting material had disappeared. The solvents were evaporated and flash chromatography (1:1 EtOAc-petroleum ether) of the residue gave the disaccharide donor 8 (300 $\mathrm{mg}, 0.29 \mathrm{mmol}, 83 \%$ ), which was used directly in the glycosylation reaction; ${ }^{1} \mathrm{H}$ NMR $\left(\mathrm{CDCl}_{3}\right): \delta 8.68(\mathrm{~s}, 1 \mathrm{H}, \mathrm{NH}), 8.12-7.16(\mathrm{~m}$, $25 \mathrm{H}, \mathrm{Bz}-\mathrm{H}), 6.42\left(\mathrm{~d}, J_{1,2} 1.8 \mathrm{~Hz}, \mathrm{H}-1\right), 6.02$ $\left(\mathrm{dd}, 1 \mathrm{H}, J_{2,3} 3.5 \mathrm{~Hz}, J_{3,4} 9.2 \mathrm{~Hz}, \mathrm{H}-3\right), 5.87$ $\left(\mathrm{dd}, 1 \mathrm{H}, J_{1,2} 1.8 \mathrm{~Hz}, J_{2,3} 3.5 \mathrm{~Hz}, \mathrm{H}-2\right), 5.67$ $\left(\mathrm{dd}, 1 \mathrm{H}, J_{2^{\prime}, 3^{\prime}} 3.2 \mathrm{~Hz}, J_{3^{\prime}, 4^{\prime}} 9.8 \mathrm{~Hz}, \mathrm{H}-3^{\prime}\right), 5.56$ (dd, $\left.1 \mathrm{H}, J_{1^{\prime}, 2^{\prime}} 1.7 \mathrm{~Hz}, J_{2^{\prime}, 3^{\prime}} 3.2 \mathrm{~Hz}, \mathrm{H}-2^{\prime}\right), 5.45$ $\left(\mathrm{t}, 1 \mathrm{H}, J 9.8 \mathrm{~Hz}, \mathrm{H}-4^{\prime}\right), 5.20\left(\mathrm{~d}, 1 \mathrm{H}, J_{1^{\prime} 2^{\prime}} 1.7\right.$ $\left.\mathrm{Hz}, \mathrm{H}-1^{\prime}\right), 5.02\left(\mathrm{dd}, 1 \mathrm{H}, J_{5,6 \mathrm{a}} 0.9 \mathrm{~Hz}, J_{6 \mathrm{a}, 6 \mathrm{~b}}\right.$ $12.4 \mathrm{~Hz}, \mathrm{H}-6 \mathrm{a}), 4.66\left(\mathrm{dd}, 1 \mathrm{H}, J_{5,6 \mathrm{~b}} 2.0 \mathrm{~Hz}\right.$, $\left.J_{6 \mathrm{a}, 6 \mathrm{~b}} 12.4 \mathrm{~Hz}, \mathrm{H}-6 \mathrm{~b}\right), 4.60$ (t, $1 \mathrm{H}, J 9.2 \mathrm{~Hz}$, $\mathrm{H}-4), 4.59-4.47$ (m, $1 \mathrm{H}, \mathrm{H}-5), 4.12-3.96$ (m, $\left.1 \mathrm{H}, \mathrm{H}-5^{\prime}\right), 2.10\left(\mathrm{~s}, 3 \mathrm{H}, \mathrm{CH}_{3} \mathrm{CO}\right), 0.87$ (d, 3 $\mathrm{H}, J 6.2 \mathrm{~Hz}, \mathrm{CH}_{3}$ ).

Allyl 4-O-benzoyl- $\alpha$-L-rhamnopyranoside(11). - Compound $\mathbf{1 1}$ was obtained as crystals from hydrolysis of $\mathbf{1 0}$, which was prepared from benzoylation of $\mathbf{9}$, according to the literature [11].

Allyl 4-O-(2,3-di-O-acetyl-4-O-benzoyl- $\alpha-\mathrm{L}-$ rhamnopyranosyl) - 2,3,6- tri - O - benzoyl - $\alpha$ - Dmannopyranoside (13). - Acetylation of compound $11(6.5 \mathrm{~g}, 21 \mathrm{mmol})$ with $\mathrm{Ac}_{2} \mathrm{O}(5 \mathrm{~mL})$ in pyridine $(10 \mathrm{~mL})$ furnished allyl 2,3-di- $O$ acetyl-4 - $O$-benzoyl- $\alpha$ - L - rhamnopyranoside 
quantitatively. Deallylation with $\mathrm{PdCl}_{2}(1 / 20$ $\mathrm{g} / \mathrm{g}$ ) in $\mathrm{MeOH}$ furnished a mixture of 2,3-di$O$-acetyl-4- $O$-benzoyl- $\alpha, \beta$-L-rhamnopyranose. Subsequent treatment with trichloroacetonitrile $\left(\mathrm{Cl}_{3} \mathrm{CCN}, 3\right.$ equiv) and 1,8-diazabicyclo[5,4,0]undec-7-ene (DBU, 0.25 equiv) at $0{ }^{\circ} \mathrm{C}$ in anhyd $\mathrm{CH}_{2} \mathrm{Cl}_{2}$ gave, after flash chromatography, the trichloroacetimidate donor 12 (595 mg, overall yield $72 \%$ for two steps), which was used directly in the glycosylation reaction [8]. A mixture of the donor $12(595$ $\mathrm{mg}, 1.19 \mathrm{mmol})$ and the acceptor $1(535 \mathrm{mg}$, $1.0 \mathrm{mmol})$ in anhyd $\mathrm{CH}_{2} \mathrm{Cl}_{2}(20 \mathrm{~mL})$ containing $4 \AA$ activated molecular sieves $(1 \mathrm{~g})$ was stirred under $\mathrm{N}_{2}$ for $1 \mathrm{~h}$ at $\mathrm{rt}$ and then cooled to $-40{ }^{\circ} \mathrm{C}$. Trimethylsilyl triflate $\left(\mathrm{Me}_{3} \mathrm{SiOTf}\right.$, $20 \mu \mathrm{L}, 0.1$ equiv) was added and the mixture was stirred under $\mathrm{N}_{2}$ below $-30{ }^{\circ} \mathrm{C}$ for 40 min. TLC showed the starting material had disappeared. Triethylamine $(25 \mu \mathrm{L})$ was added and the reaction mixture was handled as described for the preparation of $\mathbf{6}$. Flash chromatography (1:2 EtOAc-petroleum ether) of the residue gave the disaccharide $13(745 \mathrm{mg}$, $0.86 \mathrm{mmol}, 86 \%)$ as a colorless syrup; $[\alpha]_{\mathrm{D}}^{20}-$ $24^{\circ}\left(c 1.5, \mathrm{CHCl}_{3}\right) ;{ }^{1} \mathrm{H}$ NMR $\left(\mathrm{CDCl}_{3}\right): \delta 8.16-$ $7.04(\mathrm{~m}, 8 \mathrm{H}, \mathrm{Bz}-\mathrm{H}), 7.69(\mathrm{~m}, 12 \mathrm{H}, \mathrm{Bz}-\mathrm{H})$, 6.09-5.86 (m, $\left.1 \mathrm{H}, \mathrm{CH}_{2}=\mathrm{CH}-\mathrm{CH}_{2}-\right), 5.85$ $\left(\mathrm{dd}, 1 \mathrm{H}, J_{2,3} 3.7 \mathrm{~Hz}, J_{3,4} 9.3 \mathrm{~Hz}, \mathrm{H}-3\right), 5.62$ $\left(\mathrm{dd}, 1 \mathrm{H}, J_{2,3} 3.7 \mathrm{~Hz}, J_{1,2} 1.8 \mathrm{~Hz}, \mathrm{H}-2\right), 5.42$ (dd, $\left.1 \mathrm{H}, J_{2^{\prime}, 3^{\prime}} 3.8 \mathrm{~Hz}, J_{1^{\prime}, 2^{\prime}} 1.0 \mathrm{~Hz}, \mathrm{H}-2^{\prime}\right), 5.41$ $\left(\mathrm{dd}, 1 \mathrm{H}, J_{2^{\prime}, 3^{\prime}} 3.5 \mathrm{~Hz}, J_{3^{\prime}, 4^{\prime}} 9.8 \mathrm{~Hz}, \mathrm{H}-3^{\prime}\right)$, 5.35-5.22 (m, $\left.2 \mathrm{H}, \mathrm{CH}_{2}=\mathrm{CH}-\mathrm{CH}_{2}-\right), 5.18$ (t, $\left.1 \mathrm{H}, J 9.8 \mathrm{~Hz}, \mathrm{H}-4^{\prime}\right), 5.03\left(\mathrm{~d}, 1 \mathrm{H}, J_{1,2} 1.8 \mathrm{~Hz}\right.$, $\mathrm{H}-1), 5.00\left(\mathrm{~d}, 1 \mathrm{H}, J_{1^{\prime}, 2^{\prime}} 1.0 \mathrm{~Hz}, \mathrm{H}-1^{\prime}\right), 4.91(\mathrm{dd}$, $\left.1 \mathrm{H}, J_{5,6 \mathrm{a}} 2.0 \mathrm{~Hz}, J_{6 \mathrm{a}, 6 \mathrm{~b}} 12.0 \mathrm{~Hz}, \mathrm{H}-6 \mathrm{a}\right), 4.64$ $\left(\mathrm{dd}, 1 \mathrm{H}, J_{5,6 \mathrm{~b}} 3.6 \mathrm{~Hz}, J_{6 \mathrm{a}, 6 \mathrm{~b}} 12.0 \mathrm{~Hz}, \mathrm{H}-6 \mathrm{~b}\right)$, $4.50\left(\mathrm{t}, 1 \mathrm{H}, J_{3.4} 9.3 \mathrm{~Hz}, J_{4,5} 9.3 \mathrm{~Hz}, \mathrm{H}-4\right)$, 4.39-4.06 (m, $\left.3 \mathrm{H}, \mathrm{H}-5, \mathrm{CH}_{2}=\mathrm{CH}-\mathrm{CH}_{2}-\right)$, 4.00-3.84 (m, $\left.1 \mathrm{H}, \mathrm{H}-5^{\prime}\right), 2.09,1.88,(2 \mathrm{~s}, 6 \mathrm{H}$, $\left.2 \mathrm{CH}_{3} \mathrm{CO}\right), 0.73\left(\mathrm{~d}, 3 \mathrm{H}, J 6.7 \mathrm{~Hz}, \mathrm{CH}_{3}\right)$. Anal. Calcd for $\mathrm{C}_{47} \mathrm{H}_{46} \mathrm{O}_{16}: \mathrm{C}, 65.12 ; \mathrm{H}, \quad 5.35$. Found: C, 65.41; H, 5.27.

Allyl 4-O-(4-O-benzoyl- $\alpha$-L-rhamnopyranosyl)-2,3,6-tri-O - benzoyl - $\alpha$ - D - mannopyranoside (14). - A methanolic solution [12] of $\mathrm{HCl}(1.04 \mathrm{~N}, 9 \mathrm{~mL})$, prepared by adding acetyl chloride $(0.8 \mathrm{~mL})$ to freshly distilled $\mathrm{MeOH}(10 \mathrm{~mL})$, was added to a solution of the disaccharide $13(710 \mathrm{mg}, 0.82 \mathrm{mmol})$ in freshly distilled $\mathrm{MeOH}(6 \mathrm{~mL})$ and the solution was stirred at $48-54^{\circ} \mathrm{C}$. The reaction was quenched when TLC (2:1 petroleum etherEtOAc) showed $14\left(R_{f} 0.29\right)$ was the sole product. The solution was cooled to $0{ }^{\circ} \mathrm{C}$ and pyridine $(1.5 \mathrm{~mL})$ was added dropwise to neutralize the acid. The solution was concentrated to a semi-solid residue that was dissolved in $\mathrm{CH}_{2} \mathrm{Cl}_{2}(50 \mathrm{~mL})$ and washed sequentially with $\mathrm{M} \mathrm{HCl}(20 \mathrm{~mL})$, satd aq $\mathrm{NaHCO}_{3}(20 \mathrm{~mL})$, and $\mathrm{H}_{2} \mathrm{O}(20 \mathrm{~mL})$. The aq washings were re-extracted with $\mathrm{CH}_{2} \mathrm{Cl}_{2}(30 \mathrm{~mL})$ and the combined organic solutions were dried and concentrated. Gradient flash chromatography (1:1-1.5:1 EtOAc-petroleum ether) of the residue gave the diol $14(540 \mathrm{mg}, 0.69 \mathrm{mmol}$, $84 \%)$ as a colorless syrup; $[\alpha]_{\mathrm{D}}^{20}-34.2^{\circ}(c 1.2$, $\left.\mathrm{CHCl}_{3}\right) ;{ }^{1} \mathrm{H}$ NMR $\left(\mathrm{CDCl}_{3}\right): \delta 8.16-7.79(\mathrm{~m}, 8$ $\mathrm{H}, J 8.4 \mathrm{~Hz}, \mathrm{Bz}-\mathrm{H}), 7.66-7.23(\mathrm{~m}, 12 \mathrm{H}$, $\mathrm{Bz}-\mathrm{H}), 6.07-5.86\left(\mathrm{~m}, 1 \mathrm{H}, \mathrm{CH}_{2}=\mathrm{CH}-\mathrm{CH}_{2}-\right.$ ), $5.84\left(\mathrm{dd}, 1 \mathrm{H}, J_{2,3} 3.6 \mathrm{~Hz}, J_{3,4} 9.7 \mathrm{~Hz}, \mathrm{H}-3\right)$, $5.60\left(\mathrm{dd}, 1 \mathrm{H}, J_{1,2} 1.7 \mathrm{~Hz}, J_{2,3} 3.6 \mathrm{~Hz}, \mathrm{H}-2\right)$, $5.42-5.22\left(\mathrm{~m}, 2 \mathrm{H}, \mathrm{CH}_{2}=\mathrm{CH}-\mathrm{CH}_{2}-\right), 5.06(\mathrm{~d}$, $\left.1 \mathrm{H}, J_{1.2} 1.7 \mathrm{~Hz}, \mathrm{H}-1\right), 5.01\left(\mathrm{~d}, 1 \mathrm{H}, J_{1^{\prime}, 2^{\prime}} 0.8\right.$ $\mathrm{Hz}, \mathrm{H}-\mathrm{1}^{\prime}$ ), 4.93 (t, $\left.1 \mathrm{H}, J 9.7 \mathrm{~Hz}, \mathrm{H}-4^{\prime}\right), 4.77$ $\left(\mathrm{dd}, 1 \mathrm{H}, J_{5,6 \mathrm{a}} 1.8 \mathrm{~Hz}, J_{6 \mathrm{a}, 6 \mathrm{~b}} 12.3 \mathrm{~Hz}, \mathrm{H}-6 \mathrm{a}\right)$, $4.16\left(\mathrm{dd}, 1 \mathrm{H}, J_{5,6 \mathrm{~b}} 3.2 \mathrm{~Hz}, J_{6 \mathrm{a}, 6 \mathrm{~b}} 12.3 \mathrm{~Hz}\right.$, H-6b), 4.50 (t, $1 \mathrm{H}, J 9.7 \mathrm{~Hz}, \mathrm{H}-4), 4.36-4.08$ $\left(\mathrm{m}, 3 \mathrm{H}, \mathrm{H}-5, \mathrm{CH}_{2}=\mathrm{CH}-\mathrm{CH}_{2}-\right), 4.04(\mathrm{dd}, 1$ $\left.\mathrm{H}, J_{1^{\prime} 2^{\prime}} 0.8 \mathrm{~Hz}, J_{2^{\prime}, 3^{\prime}} 3.4 \mathrm{~Hz}, \mathrm{H}-2^{\prime}\right), 3.94(\mathrm{dd}, 1$ $\left.\mathrm{H}, J_{2^{\prime}, 3^{\prime}} 3.4 \mathrm{~Hz}, J_{3^{\prime}, 4^{\prime}} 9.7 \mathrm{~Hz}, \mathrm{H}-4^{\prime}\right), 3.93-3.78$ $\left(\mathrm{m}, 1 \mathrm{H}, \mathrm{H}-5^{\prime}\right), 0.74\left(\mathrm{~d}, 3 \mathrm{H}, J 6.7 \mathrm{~Hz}, \mathrm{CH}_{3}\right)$. Anal. Calcd for $\mathrm{C}_{43} \mathrm{H}_{42} \mathrm{O}_{14}$ : C, 65.98; H, 5.41. Found: C, 65.70; H, 5.54.

Allyl 4-O-[2-O-acetyl-3-O-(p-methoxybenzyl) - 4-O - benzoyl - $\alpha$ - L - rhamnopyranosyl]- 2,3, 6-tri-O-benzoyl- $\alpha$-D-mannopyranoside (15).To a solution of $14(500 \mathrm{mg}, 0.64 \mathrm{mmol})$ in $\mathrm{MeOH}(5 \mathrm{~mL})$ was added dibutyltin oxide (160 mg, 1 equiv) and the mixture was refluxed. After the mixture became clear, heating was continued for $1 \mathrm{~h}$, and the stannylene complex was obtained as a white foamy residue after evaporation of the methanol under diminished pressure. To the residue were added toluene $(6 \mathrm{~mL})$, tetrabutylammonium iodide (238 mg, 1 equiv), and $p$-methoxybenzyl chloride (120 $\mu \mathrm{L}, 1.5$ equiv), and the mixture was stirred for $20 \mathrm{~h}$ at $60-70{ }^{\circ} \mathrm{C}$. The reaction was monitored by TLC using 1:2 EtOAc-petroleum ether as the developing sol- 
vent. After completion of the alkylation [13], the solvent was evaporated under diminished pressure, and the residue was subjected to column chromatography on silica gel with $1: 2$ EtOAc-petroleum ether as the eluent. Acetylation of the resulting compound was carried out quantitatively with $\mathrm{Ac}_{2} \mathrm{O}$-pyridine, and the reaction mixture was poured into ice water $(10 \mathrm{~mL})$ and extracted with $\mathrm{CH}_{2} \mathrm{Cl}_{2}(10 \mathrm{~mL})$. The aq washings were re-extracted with $\mathrm{CH}_{2} \mathrm{Cl}_{2}(5 \mathrm{~mL})$ and the combined organic solutions were washed sequentially with $5 \%$ aq HOAc $(10 \mathrm{~mL})$, satd aq $\mathrm{NaHCO}_{3}(10 \mathrm{~mL})$, and aq $\mathrm{NaCl}(10 \mathrm{~mL})$. The organic phase was dried and concentrated under reduced pressure, and the pure, colorless syrupy product $15(450 \mathrm{mg}, 75 \%)$ was obtained after separation on a silica gel column with 1:3 EtOAcpetroleum ether as eluent; $[\alpha]_{\mathrm{D}}^{20}-54.1^{\circ}(c) 3.7$, $\left.\mathrm{CHCl}_{3}\right) ;{ }^{1} \mathrm{H} \mathrm{NMR}\left(\mathrm{CDCl}_{3}\right): \delta 8.10-7.73(4 \mathrm{~d}$, $8 \mathrm{H}, J 8.6 \mathrm{~Hz}, \mathrm{Bz}-\mathrm{H}), 7.68-7.24(\mathrm{~m}, 12 \mathrm{H}$, $\mathrm{Bz}-\mathrm{H}), 7.05,6.64(2 \mathrm{~d}, 4 \mathrm{H}, J 9.1 \mathrm{~Hz}, \mathrm{pMB}-$ $\mathrm{H}), 6.09-5.86\left(\mathrm{~m}, 1 \mathrm{H}, \mathrm{CH}_{2}=\mathrm{CH}-\mathrm{CH}_{2}-\right), 5.84$ (dd, $\left.1 \mathrm{H}, J_{2,3} 2.3 \mathrm{~Hz}, J_{3,4} 9.3 \mathrm{~Hz}, \mathrm{H}-3\right), 5.61$ $\left(\mathrm{dd}, 1 \mathrm{H}, J_{1,2} 1.7 \mathrm{~Hz}, J_{2,3} 2.3 \mathrm{~Hz}, \mathrm{H}-2\right), 5.44-$ $5.24\left(\mathrm{~m}, 2 \mathrm{H}, \mathrm{CH}_{2}=\mathrm{CH}-\mathrm{CH}_{2}-\right), 5.25(\mathrm{dd}, 1 \mathrm{H}$, $\left.J_{1^{\prime}, 2^{\prime}} 1.2 \mathrm{~Hz}, J_{2^{\prime}, 3^{\prime}} 3.6 \mathrm{~Hz}, \mathrm{H}-2^{\prime}\right), 5.13(\mathrm{t}, 1 \mathrm{H}, J$ $\left.9.8 \mathrm{~Hz}, \mathrm{H}-4^{\prime}\right), 5.05\left(\mathrm{~d}, 1 \mathrm{H}, J_{1,2} 1.7 \mathrm{~Hz}, \mathrm{H}-1\right)$, $5.03\left(\mathrm{~d}, 1 \mathrm{H}, J_{1^{\prime}, 2^{\prime}} 1.2 \mathrm{~Hz}, \mathrm{H}-1^{\prime}\right), 4.94(\mathrm{dd}, 1 \mathrm{H}$, $\left.J_{5,6 \mathrm{a}} 1.2 \mathrm{~Hz}, J_{6 \mathrm{a} .6 \mathrm{~b}} 12.3 \mathrm{~Hz}, \mathrm{H}-6 \mathrm{a}\right), 4.52(\mathrm{t}, 1 \mathrm{H}$, $J 9.3 \mathrm{~Hz}, \mathrm{H}-4), 4.51\left(\mathrm{dd}, 1 \mathrm{H}, J_{5,6 \mathrm{~b}} 5.6 \mathrm{~Hz}\right.$, $\left.J_{6 \mathrm{a}, 6 \mathrm{~b}} 12.3 \mathrm{~Hz}, \mathrm{H}-6 \mathrm{~b}\right), 4.49,4.37$ (ABq, $2 \mathrm{H},{ }^{2} J$ $11.6 \mathrm{~Hz}, \mathrm{pMBCH})_{2}, 4.38-4.07$ (m, $4 \mathrm{H}, \mathrm{H}-5$, $\left.5^{\prime}, \mathrm{CH}_{2}=\mathrm{CH}-\mathrm{CH}_{2}-\right), 3.84\left(\mathrm{dd}, 1 \mathrm{H}, J_{2^{\prime}, 3^{\prime}} 3.6\right.$ $\left.\mathrm{Hz}, J_{3^{\prime}, 4^{\prime}} 9.8 \mathrm{~Hz}, \mathrm{H}-3^{\prime}\right), 3.74\left(\mathrm{~s}, 3 \mathrm{H}, \mathrm{OCH}_{3}\right)$, $2.09\left(\mathrm{~s}, 3 \mathrm{H}, \mathrm{CH}_{3} \mathrm{CO}\right), 0.74(\mathrm{~d}, 3 \mathrm{H}, J 6.8 \mathrm{~Hz}$, $\mathrm{CH}_{3}$ ). Anal. Calcd for $\mathrm{C}_{53} \mathrm{H}_{52} \mathrm{O}_{16}: \mathrm{C}, 67.36 ; \mathrm{H}$, 5.55. Found: $\mathrm{C}, 67.50 ; \mathrm{H}, 5.58$.

Allyl 4-O-(2-O-acetyl-4-O-benzoyl- $\alpha$-Lrhamnopyranosyl) - 2,3,6- tri - O - benzoyl - $\alpha$ - Dmannopyranoside (16) by direct selective 3'deacetylation of 13. - A methanolic solution of $\mathrm{HCl}(1.04 \mathrm{~N}, 5 \mathrm{~mL})$, prepared by adding acetyl chloride $(0.8 \mathrm{~mL})$ to freshly distilled $\mathrm{MeOH}(10 \mathrm{~mL})$, was added to a solution of the disaccharide $13(340 \mathrm{mg}, 0.39 \mathrm{mmol})$ in freshly distilled $\mathrm{MeOH}(3.3 \mathrm{~mL})$ and the solution was stirred at $45-50{ }^{\circ} \mathrm{C}$ for about $1 \mathrm{~h}$. The reaction was monitored by TLC (2:1 petroleum ether-EtOAc), and the reaction was quenched when TLC showed that the maximum amount of major product $\left(R_{f} 0.65\right)$ [minor product $\left(R_{f} 0.29\right)$, starting material $\left(R_{f}\right.$ $0.82)$ ] had been reached. The solution was cooled to $0{ }^{\circ} \mathrm{C}$ and pyridine $(1 \mathrm{~mL})$ was added dropwise to neutralize the acid. The solution was concentrated to a semi-solid residue that was dissolved in $\mathrm{CH}_{2} \mathrm{Cl}_{2}(10 \mathrm{~mL})$ and washed sequentially with $\mathrm{M} \mathrm{HCl}(10 \mathrm{~mL})$, satd aq $\mathrm{NaHCO}_{3}(10 \mathrm{~mL})$, and $\mathrm{H}_{2} \mathrm{O}(10 \mathrm{~mL})$. The aq washings were re-extracted with $\mathrm{CH}_{2} \mathrm{Cl}_{2}(10$ $\mathrm{mL}$ ) and the combined organic solutions were dried and concentrated. Gradient flash chromatography $(1: 2-1: 1-1.5: 1 \quad$ EtOAc-petroleum ether) of the residue gave $\mathbf{1 6}(206 \mathrm{mg}$, $0.25 \mathrm{mmol}, 65 \%)$ as a colorless syrup; $[\alpha]_{\mathrm{D}}^{20}-$ $80^{\circ}\left(\mathrm{c} 1.2, \mathrm{CHCl}_{3}\right) ;{ }^{1} \mathrm{H}$ NMR $\left(\mathrm{CDCl}_{3}\right): \delta 8.20-$ $7.80(\mathrm{~m}, 8 \mathrm{H}, \mathrm{Bz}-\mathrm{H}), 7.68-7.26(\mathrm{~m}, 12 \mathrm{H}$, $\mathrm{Bz}-\mathrm{H}), 6.07-5.87\left(\mathrm{~m}, 1 \mathrm{H}, \mathrm{CH}_{2}=\mathrm{CH}-\mathrm{CH}_{2}-\right.$ ), $5.85\left(\mathrm{dd}, 1 \mathrm{H}, J_{3,4} 9.7 \mathrm{~Hz}, J_{2,3} 3.6 \mathrm{~Hz}, \mathrm{H}-3\right)$, $5.61\left(\mathrm{dd}, 1 \mathrm{H}, J_{1,2} 1.7 \mathrm{~Hz}, J_{2,3} 3.2 \mathrm{~Hz}, \mathrm{H}-2\right)$, 5.42-5.22 (m, $\left.2 \mathrm{H}, \mathrm{CH}_{2}=\mathrm{CH}-\mathrm{CH}_{2}-\right), 5.09(\mathrm{~d}$, $\left.1 \mathrm{H}, J_{1,2} 1.7 \mathrm{~Hz}, \mathrm{H}-1\right), 5.05\left(\mathrm{dd}, 1 \mathrm{H}, J_{1^{\prime}, 2^{\prime}} 1.2\right.$ $\left.\mathrm{Hz}, J_{2^{\prime}, 3^{\prime}} 3.3 \mathrm{~Hz}, \mathrm{H}-2^{\prime}\right), 5.03\left(\mathrm{~d}, 1 \mathrm{H}, J_{1^{\prime}, 2^{\prime}} 1.2\right.$ $\left.\mathrm{Hz}, \mathrm{H}^{\prime} 1^{\prime}\right), 4.99\left(\mathrm{dd}, 1 \mathrm{H}, J_{5,6 \mathrm{a}} 1.2 \mathrm{~Hz}, J_{6 \mathrm{a}, 6 \mathrm{~b}}\right.$ $12.2 \mathrm{~Hz}, \mathrm{H}-6 \mathrm{a}), 4.94$ (t, $\left.1 \mathrm{H}, J 9.7 \mathrm{~Hz}, \mathrm{H}-4^{\prime}\right)$, $4.59\left(\mathrm{dd}, 1 \mathrm{H}, J_{5,6 \mathrm{~b}} 3.1 \mathrm{~Hz}, J_{6 \mathrm{a}, 6 \mathrm{~b}} 12.2 \mathrm{~Hz}\right.$, H-6b), 4.53 (t, $1 \mathrm{H}, J 9.7 \mathrm{~Hz}, \mathrm{H}-4), 4.36-4.05$ (m, $4 \mathrm{H}, \mathrm{H}-3^{\prime}, 5, \mathrm{CH}_{2}=\mathrm{CH}-\mathrm{CH}_{2}-$ ), 3.95-3.78 (m, $\left.1 \mathrm{H}, \mathrm{H}-5^{\prime}\right), 2.08$ (s, $3 \mathrm{H}, \mathrm{CH}_{3} \mathrm{CO}$ ), 0.73 (s, $3 \mathrm{H}, J 6.8 \mathrm{~Hz}, \mathrm{CH}_{3}$ ). Anal. Calcd for $\mathrm{C}_{45} \mathrm{H}_{44} \mathrm{O}_{15}: \mathrm{C}, 65.53 ; \mathrm{H}, 5.38$. Found: $\mathrm{C}, 65.40$; $\mathrm{H}, 5.27$.

Allyl 4-O-(2-O-acetyl-4-O-benzoyl- $\alpha-\mathrm{L}-$ rhamnopyranosyl) - 2,3,6- tri-O - benzoyl $-\alpha-\mathrm{D}$ mannopyranoside (16). - Solid 2,3-dichloro5,6-dicyano-1,4-benzoquinone (DDQ, $0.11 \mathrm{~g}$, $0.48 \mathrm{mmol}$ ) [14] was added with stirring at $\mathrm{rt}$ to a mixture of compound $\mathbf{1 5}(420 \mathrm{mg}, 0.44$ $\mathrm{mmol}), \mathrm{CH}_{2} \mathrm{Cl}_{2}(5 \mathrm{~mL})$, and water $(0.25 \mathrm{~mL})$. Stirring was continued for $6 \mathrm{~h}$, when TLC (1:2 EtOAc-petroleum ether) showed complete conversion of the starting material to a slower moving product. $\mathrm{CH}_{2} \mathrm{Cl}_{2}(5 \mathrm{~mL})$ was added, the resulting solution was washed with aq $\mathrm{Na}_{2} \mathrm{~S}_{2} \mathrm{O}_{3}$, dried, and concentrated, and the residue was chromatographed to give $\mathbf{1 6}$ (318 $\mathrm{mg}, 88 \%$ ). Its ${ }^{1} \mathrm{H}$ NMR spectrum was the same as that of the product obtained from the direct selective 3'-deacetylation of $\mathbf{1 3}$. 
Allyl 4-O-\{3-O-[4-O-(2-O-acetyl-3,4-di-Obenzoyl - $\alpha$ - L - rhamnopyranosyl) - 2,3,6- tri - Obenzoyl- $\alpha-\mathrm{D}$-mannopyranosyl]-2-O-acetyl-4$\mathrm{O}$ - benzoyl - $\alpha$ - L - rhamnopyranosyl $\}$ - 2,3,6 - triO-benzoyl- $\alpha$-D-mannopyranoside

(17). - A mixture of the disaccharide donor $8(289 \mathrm{mg}$, $0.28 \mathrm{mmol}$ ) and the disaccharide acceptor $\mathbf{1 6}$ (230 mg, $0.28 \mathrm{mmol}$ ) in anhyd $\mathrm{CH}_{2} \mathrm{Cl}_{2}(15 \mathrm{~mL})$ containing $4 \AA$ activated molecular sieves ( $1 \mathrm{~g}$ ) was stirred under $\mathrm{N}_{2}$ for $1 \mathrm{~h}$ at $\mathrm{rt}$ and then cooled to $-40{ }^{\circ} \mathrm{C}$. Trimethylsilyl triflate ( $\mathrm{Me}_{3} \mathrm{SiOTf}, 5 \mu \mathrm{L}, 0.1$ equiv) was added and the mixture was stirred under $\mathrm{N}_{2}$ below $-30{ }^{\circ} \mathrm{C}$ for another $40 \mathrm{~min}$. TLC showed the starting material had disappeared. Triethylamine (5 $\mu \mathrm{L})$ was added and the mixture was treated as described for the preparation of $\mathbf{6}$. Flash chromatography (2:1 EtOAc-petroleum ether) of the residue gave the tetrasaccharide 17 (360 $\mathrm{mg}, 0.21 \mathrm{mmol}, 75 \%$ ) as a colorless syrup; $[\alpha]_{\mathrm{D}}^{20}-42.6^{\circ}\left(c 1.0, \mathrm{CHCl}_{3}\right) ;{ }^{1} \mathrm{H} \mathrm{NMR}\left(\mathrm{CDCl}_{3}\right)$ : $\delta 8.20-7.74(\mathrm{~m}, 18 \mathrm{H}, \mathrm{Bz}-\mathrm{H}), 7.70-7.16(\mathrm{~m}, 27$ $\mathrm{H}, \mathrm{Bz}-\mathrm{H}), 6.08-5.86\left(\mathrm{~m}, 1 \mathrm{H}, \mathrm{CH}_{2}=\mathrm{CH}-\right.$ $\left.\mathrm{CH}_{2}-\right), 5.87\left(\mathrm{dd}, 1 \mathrm{H}, J_{2 \mathrm{~A}, 3 \mathrm{~A}} 3.7 \mathrm{~Hz}, J_{3 \mathrm{~A}, 4 \mathrm{~A}} 9.8\right.$ $\mathrm{Hz}, \mathrm{H}-3 \mathrm{~A}), 5.64$ (dd, $1 \mathrm{H}, J_{1 \mathrm{~A}, 2 \mathrm{~A}} 1.7 \mathrm{~Hz}, J_{2 \mathrm{~A}, 3 \mathrm{~A}}$ $3.7 \mathrm{~Hz}, \mathrm{H}-2 \mathrm{~A}), 5.58-5.21(\mathrm{~m}, 8 \mathrm{H}, \mathrm{H}-2 \mathrm{~B}, 2 \mathrm{C}$, 2D, 3C, 3D, 4D, $\mathrm{CH}_{2}=\mathrm{CH}-\mathrm{CH}_{2}-$ ), 5.14, 5.08, 5.04, 4.99 (4 d, 4 H, H-1A, 1B, 1C, 1D), 4.83 $\left(\mathrm{dd}, 1 \mathrm{H}, J_{5 \mathrm{~A}, 6 \mathrm{~A}} 0.7 \mathrm{~Hz}, J_{6 \mathrm{~A}, 6 \mathrm{~A}} 12.7 \mathrm{~Hz}, \mathrm{H}-6 \mathrm{~A}\right)$, 4.65-3.70 (m, $12 \mathrm{H}, \mathrm{H}-3 \mathrm{~B}, 4 \mathrm{~A}, 4 \mathrm{C}, 5 \mathrm{~A}, 5 \mathrm{~B}, 5 \mathrm{C}$, 5D, 6A, 6C, 6C, $\left.\mathrm{CH}_{2}=\mathrm{CH}-\mathrm{CH}_{2}-\right), 2.26,2.02$ $\left(2 \mathrm{~s}, 6 \mathrm{H}, 2 \mathrm{CH}_{3} \mathrm{CO}\right), 0.85,0.72(2 \mathrm{~d}, 6 \mathrm{H}, J 6.2$ $\left.\mathrm{Hz}, 2 \mathrm{CH}_{3}\right)$. Anal. Calcd for $\mathrm{C}_{94} \mathrm{H}_{86} \mathrm{O}_{30}$ : C, 66.58; H, 5.11. Found: C, 66.59; H, 5.20.

Allyl 4-O- $\{3-\mathrm{O}-[4-\mathrm{O}-(3,4-d i-\mathrm{O}-$ benzoyl- $\alpha-\mathrm{L}-$ rhamnopyranosyl) - 2,3,6-tri - O - benzoyl - $\alpha$ - Dmannopyranosyl]-4-benzoyl- $\alpha$-L-rhamnopyranosyl\} - 2,3,6-tri-O - benzoyl- $\alpha$ - D - mannopyranoside (18). - A methanolic solution of $\mathrm{HCl}$ (1.04 N, $2.2 \mathrm{~mL})$ prepared by adding acetyl chloride $(0.8 \mathrm{~mL})$ to freshly distilled $\mathrm{MeOH}(10$ $\mathrm{mL})$, was added to a solution of $17(320 \mathrm{mg}$, $0.19 \mathrm{mmol})$ in freshly distilled $\mathrm{MeOH}(1.5 \mathrm{~mL})$ and the solution was stirred at $48-52{ }^{\circ} \mathrm{C}$ for 1.5 $\mathrm{h}$ to give 18 as a syrup (260 mg, $0.16 \mathrm{mmol}$, $84 \%)$ after flash chromatography; $[\alpha]_{\mathrm{D}}^{20}-49.4^{\circ}$ (c $\left.0.7 \mathrm{CHCl}_{3}\right) ;{ }^{1} \mathrm{H} \mathrm{NMR}\left(\mathrm{CDCl}_{3}\right): \delta 8.17-7.80$ $(\mathrm{m}, 18 \mathrm{H}, \mathrm{Bz}-\mathrm{H}), 7.68-7.20(\mathrm{~m}, 27 \mathrm{H}, \mathrm{Bz}-\mathrm{H})$, 6.07-5.82 (m, 1 H, CH $\left.\mathrm{CH}_{2} \mathrm{CH}-\mathrm{CH}_{2}-\right), 5.86(\mathrm{dd}$, $\left.1 \mathrm{H}, J_{2 \mathrm{~A}, 3 \mathrm{~A}} 3.2 \mathrm{~Hz}, J_{3 \mathrm{~A}, 4 \mathrm{~A}} 9.8 \mathrm{~Hz}, \mathrm{H}-3 \mathrm{~A}\right)$,
5.68-5.02 (m, 8 H, H-2A, 2C, 3C, 3D, 4D, 6A, $\left.\mathrm{CH}_{2}=\mathrm{CH}-\mathrm{CH}_{2}-\right), 5.22,5.12,5.05,5.04(4 \mathrm{~d}, 4$ $\mathrm{H}, \mathrm{H}-1 \mathrm{~A}, 1 \mathrm{~B}, 1 \mathrm{C}, 1 \mathrm{D}), 4.89-3.80(\mathrm{~m}, 14 \mathrm{H}$, H-2B, 2D, 3B, 4A, 4C, 5A, 5B, 5C, 5D, 6A, 6C, 6C, $\left.\mathrm{CH}_{2}=\mathrm{CH}-\mathrm{CH}_{2}-\right), 0.81,0.76(2 \mathrm{~d}, 6 \mathrm{H}, J$ $\left.6.2 \mathrm{~Hz}, 2 \mathrm{CH}_{3}\right)$. Anal. Calcd for $\mathrm{C}_{90} \mathrm{H}_{82} \mathrm{O}_{28}: \mathrm{C}$, 67.07; H, 5.13. Found: C, 67.01; H, 5.15.

Allyl 4-O-\{2-O-[2,3,4-tri-O-acetyl- $\beta$-L- $x y l-$ opyranosyl] - 3 - O - [4 - O - (2 - O - (2,3,4 - tri - Oacetyl- $\beta$ - L-xylopyranosyl)-3,4-di-O-benzoyl$\alpha$-L-rhamnopyranosyl)-2,3,6-tri-O-benzoyl- $\alpha$ D-mannopyranosyl]-4-O-benzoyl- $\alpha$-L-rhamnopyranosyl $\}$ - 2,3,6-tri-O - benzoyl - $\alpha$ - D - mannopyranoside (19). - Coupling of 2,3,4-tri- $O$ acetyl- $\alpha$-L-xylopyranosyl bromide (128 mg, 0.3 mmol), obtained from L-xylose (purchased from Fluka) according to the standard method for the preparation of 'acetobromoglucose' [15], with the tetrasaccharide acceptor 18 (170 $\mathrm{mg}, 0.106 \mathrm{mmol})$, promoted by silver triflate (77 mg, $0.3 \mathrm{mmol})$, in dry $\mathrm{CH}_{2} \mathrm{Cl}_{2}(10 \mathrm{~mL})$ containing $4 \AA$ activated molecular sieves $(0.5$ g) under $\mathrm{N}_{2}$ was carried out at $-5^{\circ} \mathrm{C}$ for $2 \mathrm{~h}$, at the end of which time TLC showed the starting material had disappeared. Pyridine $(150 \mu \mathrm{L})$ and $10 \%$ aq $\mathrm{Na}_{2} \mathrm{~S}_{2} \mathrm{O}_{3}(1 \mathrm{~mL})$ were added to quench the reaction. The reaction mixture was dissolved in $\mathrm{CH}_{2} \mathrm{Cl}_{2}(10 \mathrm{~mL})$ and washed sequentially with $\mathrm{N} \mathrm{HCl}(10 \mathrm{~mL})$, satd aq $\mathrm{NaHCO}_{3}(10 \mathrm{~mL})$, and $\mathrm{H}_{2} \mathrm{O}(10 \mathrm{~mL})$. The aq washings were re-extracted with $\mathrm{CH}_{2} \mathrm{Cl}_{2}(5$ $\mathrm{mL}$ ) and the combined organic solutions were dried and concentrated. After flash chromatography with $3: 1$ petroleum ether-EtOAc as the eluent, hexasaccharide $19(152 \mathrm{mg}, 0.071$ mmol, 67\%) was obtained; $[\alpha]_{\mathrm{D}}^{20}+36.8^{\circ}(c 1.8$ $\left.\mathrm{CHCl}_{3}\right) ;{ }^{1} \mathrm{H}$ NMR $\left(\mathrm{CDCl}_{3}\right): \delta 8.18-7.84(\mathrm{~m}, 18$ $\mathrm{H}, \mathrm{Bz}-\mathrm{H}), 7.72-7.22(\mathrm{~m}, 27 \mathrm{H}, \mathrm{Bz}-\mathrm{H}), 6.04-$ $5.93\left(\mathrm{~m}, 1 \mathrm{H}, \mathrm{CH}_{2}=\mathrm{CH}-\mathrm{CH}_{2}-\right), 5.85(\mathrm{dd}, 1 \mathrm{H}$, $\left.J_{2 \mathrm{~A}, 3 \mathrm{~A}} 3.4 \mathrm{~Hz}, J_{3 \mathrm{~A}, 4 \mathrm{~A}} 9.6 \mathrm{~Hz}, \mathrm{H}-3 \mathrm{~A}\right), 5.74(\mathrm{dd}, 1$ $\left.\mathrm{H}, J_{1 \mathrm{~A}, 2 \mathrm{~A}} 1.7 \mathrm{~Hz}, J_{2 \mathrm{~A}, 3 \mathrm{~A}} 3.4 \mathrm{~Hz}, \mathrm{H}-2 \mathrm{~A}\right), 5.63-$ 5.59 (m, $2 \mathrm{H}, \mathrm{H}-2 \mathrm{C}, 3 \mathrm{C}), 5.39-5.20$ (m, $2 \mathrm{H}$, $\left.\mathrm{CH}_{2}=\mathrm{CH}-\mathrm{CH}_{2}-\right), 5.30-4.52(\mathrm{~m}, 17 \mathrm{H}, \mathrm{H}-1 \mathrm{~A}$, $1 \mathrm{~B}, 1 \mathrm{C}, 1 \mathrm{D}, 2 \mathrm{E}, 2 \mathrm{~F}, 3 \mathrm{D}, 3 \mathrm{E}, 3 \mathrm{~F}, 4 \mathrm{~B}, 4 \mathrm{D}, 4 \mathrm{E}$, $4 \mathrm{~F}, 6 \mathrm{~A}, 6 \mathrm{C}), 4.46-4.10(\mathrm{~m}, 13 \mathrm{H}, \mathrm{H}-1 \mathrm{E}, 1 \mathrm{~F}$, $2 \mathrm{~B}, 2 \mathrm{D}, 3 \mathrm{~B}, 4 \mathrm{~A}, 4 \mathrm{C}, 6 \mathrm{E}, 6 \mathrm{~F}, \mathrm{CH}_{2}=\mathrm{CH}-\mathrm{CH}_{2}-$ ), $4.05-3.11(\mathrm{~m}, 8 \mathrm{H}, \mathrm{H}-5 \mathrm{~A}, 5 \mathrm{~B}, 5 \mathrm{C}, 5 \mathrm{D}, 5 \mathrm{E}, 5 \mathrm{~F})$, $2.12,2.03,2.03,2.02,1.74,1.57(6 \mathrm{~s}, 18 \mathrm{H}, 6$ $\left.\mathrm{CH}_{3} \mathrm{CO}\right), 0.73$ (d, $\left.3 \mathrm{H}, J 6.2 \mathrm{~Hz}, \mathrm{CH}_{3}\right), 0.61$ (d, $\left.3 \mathrm{H}, J 6.2 \mathrm{~Hz}, \mathrm{CH}_{3}\right)$. Anal. Calcd for $\mathrm{C}_{112} \mathrm{H}_{110} \mathrm{O}_{42}$ : $\mathrm{C}, 63.21 ; \mathrm{H}$, 5.21. Found: $\mathrm{C}$, 63.10; H, 5.25. 
Allyl 4-O- $\{2-\mathrm{O}-[\beta-\mathrm{L}-x y$ lopyranosyl]-3-O-[4$\mathrm{O}$ - (2 - O - ( $\beta$ - L - xylopyranosyl $)-\alpha-\mathrm{L}$ - rhamno pyranosyl)- $\alpha-\mathrm{D}-$ mannopyranosyl]- $\alpha-\mathrm{L}-$ rhamnopyranosyl $\}-\alpha$-D-mannopyranoside (20). - The protected hexasaccharide 19 (120 mg, 0.056 mmol) was suspended in freshly distilled $\mathrm{MeOH}(10 \mathrm{~mL})$, a solution of sodium methoxide $(2 \mathrm{M}, 0.5 \mathrm{~mL})$ was added, and the mixture was stirred overnight at rt. More sodium methoxide $(0.5 \mathrm{~mL})$ was added, and the reaction was heated at $40^{\circ} \mathrm{C}$ for another $8 \mathrm{~h}$. TLC showed that the reaction was complete. The resulting solution was de-ionized with Amberlite IR-120 $\left(\mathrm{H}^{+}\right)$anion-exchange resin, filtered, and evaporated. The hexasaccharide 20 was obtained after chromatography on Sephadex G-25 $\left(\mathrm{H}_{2} \mathrm{O}\right.$ solvent) as an amorphous powder (51 mg, 97\%) after freeze-drying; $[\alpha]_{\mathrm{D}}^{20}+33.5^{\circ}$ ( c 1.8, MeOH); ESMS for $\mathrm{C}_{37} \mathrm{H}_{62} \mathrm{O}_{27}$ (938.88): $937.7[M-1]^{+} .{ }^{1} \mathrm{H} \mathrm{NMR}$ $\left(\mathrm{D}_{2} \mathrm{O}\right): \delta 6.00-5.85\left(\mathrm{~m}, 1 \mathrm{H}, \mathrm{CH}_{2}=\mathrm{CH}-\mathrm{CH}_{2}-\right.$ ), 5.32-5.17 (m, $2 \mathrm{H}, \mathrm{CH}_{2}=\mathrm{CH}-\mathrm{CH}_{2}-$ ), 5.00, 4.94, 4.94, 4.87 (4 s, 4 H, H-1A, 1B, 1C, 1D), 4.32, $4.30\left(2 \mathrm{~d}, 2 \mathrm{H}, J_{1 \mathrm{E}, 2 \mathrm{~F}} 7.6 \mathrm{~Hz}, \mathrm{H}-1 \mathrm{E}, 1 \mathrm{~F}\right)$, 4.24-4.15 (m, 2 H, $\left.\mathrm{CH}_{2}=\mathrm{CH}-\mathrm{CH}_{2}-\right)$, $1.23,1.23\left(2 \mathrm{~d}, 6 \mathrm{H}, J 4.3 \mathrm{~Hz}, \mathrm{CH}_{3}\right)$.

\section{Acknowledgements}

This work was supported by the Chinese Academy of Sciences (Project KJ952J,510) and by the National Natural Science Foundation of China (Project 29802009).

\section{References}

[1] (a) N.J. Palleroni, J.F. Bradbury, Int. J. Syst. Bacteriol., 43 (1993) 606-609. (b) R. Hugh, E. Ryschenkow, J. Gen. Microbiol., 26 (1961) 123-132. (c) J. Swings, P. De Vos, M. Van den Mooter, J. De Ley, Int. J. Syst. Bacteriol., 33 (1983) 409-413.

[2] A.M. Winn, S.G. Wilkinson, Carbohydr. Res., 298 (1997) 213-217.

[3] S. Xiong, F. Kong, C. Yang, Youji Huaxue, 14 (1994) $280-285$.

[4] R.W. Bassily, M.A. Nashed, A.S. Nematalla, Carbohydr. Res., 239 (1993) 197-207.

[5] (a) R.U. Lemieux, A.R. Morgan, Can. J. Chem., 43 (1965) 2199-2213. (b) D.R. Bundle, S. Josephson, Can. J. Chem., 57 (1979) 662-668. (c) V. Pozsgay, Carbohydr. Res., 80 (1980) 196-202.

[6] S. Saito, S. Sumita, K. Ichinose, Y. Kanda, Chem. Pharm. Bull., 41 (1993) 90-96.

[7] R.R. Schmidt, Angew. Chem., Int. Ed. Engl., 25 (1986) $212-235$.

[8] R.R. Schmidt, W. Kinzy, Adv. Carbohydr. Chem. Biochem., 50 (1994) 21-123.

[9] T. Ogawa, S. Nakabayashi, Carbohydr. Res., 93 (1981) C1-C5.

[10] J.S. Brimacobe, M.C. Cook, L.C.N. Tucker, J. Chem. Soc., (1965) 2292-2297.

[11] J.R. Marino-Albernas, V. Verez-Bencomo, L. Gonzales, C.S. Perez, Carbohydr. Res., 165 (1987) 197-206.

[12] (a) E. Petrakova, J. Schraml, Collect. Czech. Chem. Commun., 48 (1983) 877-888. (b) F.-I. Auzanneau, F. Forooghian, B.M. Pinto, Carbohydr. Res., 291 (1996) 21-41.

[13] G. Yang, F. Kong, Carbohydr. Res., 211 (1991) 179182.

[14] K. Horita, T. Yoshioka, T. Tanaka, Y. Oikawa, O. Yonemitsu, Tetrahedron, 42 (1986) 3021-3028.

[15] R.U. Lemieux, Methods Carbohydr. Chem., 2 (1963) $221-222$.

[16] T. Ziegler, E. Eckhardt, D. Keller, J. Carbohydr. Chem., 16 (1997) 719-738. 